\title{
A Novel Optical Vector Spectral Analysis Technique Employing a Limited-Bandwidth Detector
}

\author{
C. K. Madsen \\ Department of Electrical Engineering, Texas A\&M University, College Station, TX 77843, USA \\ Email:cmadsen@ee.tamu.edu
}

Received 23 April 2004; Revised 6 December 2004

\begin{abstract}
A new technique for obtaining frequency-dependent magnitude and phase information across an optical channel is presented using tunable allpass optical filters and a detector with a small bandwidth relative to the full channel width. This technique has application to optical monitoring of intersymbol interference distortions, including chromatic and polarization-mode dispersion effects, and thus can provide vector information for input to the control of adaptive optical filters. A method for generating a test signal spanning the spectrum of a modulated data signal without introducing intersymbol interference is discussed. This technique can also be used to characterize an optical pulse source and does not scale in complexity or cost as the bandwidth of the source increases.
\end{abstract}

Keywords and phrases: intersymbol interference, chromatic and polarization-mode dispersion, optical channel monitoring, optical channel estimation.

\section{INTRODUCTION}

A novel optical vector spectral analysis (OVSA) technique is proposed that measures the phase and amplitude of a signal using a detector having a limited bandwidth with respect to the full signal bandwidth. When a known signal with spectral components spanning the channel width is launched into a wavelength-division-multiplexed (WDM) optical communication system, it allows the frequency response of the system across the channel bandwidth to be determined. Given the importance of chromatic dispersion and polarizationmode dispersion (PMD) in high-bitrate optical communication systems, the OVSA can be implemented to characterize both the frequency-dependent relative phase and the signal's polarization-dependence. The goal of characterizing the channel, or system response across a limited spectral width, is to provide accurate information on signal distortion. This information may then be used as a signal quality monitor or to update an adaptive filter that compensates for distortions $[1,2]$ and subsequently allows a higher bitrate times distance product to be achieved for the system. The proposed technique also allows a periodically modulated optical source to be fully characterized.

A previous OVSA technique suggested the use of narrowband optical filters to extract sidebands equally spaced around a return-to-zero (RZ) modulated carrier [3], from which relative delay and dispersion information could be obtained without introducing any special test signals at the transmitter. One narrowband filter (NBF) was required per tone, so a minimum of three filters were required, one at the carrier and one for each sidetone. The detector bandwidth was necessarily on the order of the signal spectral width. It is desired to keep the detector bandwidth as small as possible for low cost and to minimize the complexity of the optical circuit as well. It is also desirable to arbitrarily increase the number of discrete frequencies at which measurements are made to increase the allowable PMD compensation range or baseband data rate or both. The new technique is proposed with these considerations in mind. This approach does not necessarily change the input polarization at the transmitter, unlike [4].

In this paper, we begin with a description of the optical source properties that are critical to our new OVSA method and some applicable source generation techniques. The analyzer is then described theoretically, followed by a presentation of system simulations to verify the expected performance. While the new OVSA can be used to characterize a periodic input pulse stream with an arbitrary waveform shape using a much simpler implementation that assumes a fixed input state of polarization, the explanation is given for an implementation that allows a channel with polarizationdependent loss and polarization-mode dispersion to be characterized. In the latter case, vector analysis implies characterization with respect to the phase response as in a traditional vector network analyzer as well as with respect to the vector nature of the incoming polarization. 


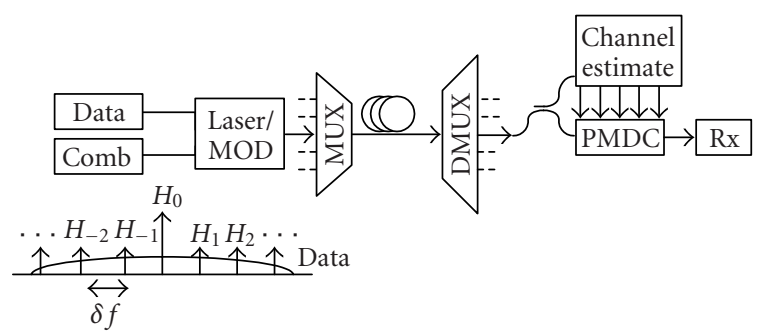

FIGURE 1: Modified transmitter consisting of a laser/modulator driven with data and test frequency comb embedded in a WDM system with a tap on the receiver side for channel estimation. The output spectrum at the transmitter is shown in the inset.

\section{OPTICAL SOURCE GENERATION}

While phase and magnitude information can be obtained from the modulated signal without modification to the transmitter [3], methods to enhance the signal-to-noise ratio (SNR) and decrease the complexity and cost of the measurement are needed. Test, or training, signals are used in wireless transmission on dedicated frequency and/or time slots to characterize the channel response. In optical communications, burst-mode transmission is unlikely to be introduced for inserting time slot monitors and frequency allocation within the spectrum of a high-bitrate signal, where we wish to measure the system response, since it could produce more distortion than we're trying to measure. Consequently, we focus on adding information to the optical signal that will minimally impact its transmission in terms of intersymbol interference (ISI). Sensitivity to nonlinearities is also important, but it is beyond the scope of this paper. It is proposed to add a test signal to the data signal in the form of a comb with known relative magnitude and phase relationships at the transmitter as shown in Figure 1, where $\delta f$ may be on the order of 2.5 to $5 \mathrm{GHz}$ for practical purposes. This provides information with a higher SNR than trying to extract the desired information from the equivalent of a pseudorandom bit sequence (PRBS). With the test comb and proposed analyzer circuit, the fast photodetectors need only have enough bandwidth to accommodate $\delta f$ and not the whole signal bandwidth!

The tones are assumed to be equally spaced in this paper. Equally spaced tones are easy to generate using a steprecovery diode. For completeness, it is noted that chirped tones, whereby each pair of adjacent tones has a slightly different spacing, would produce a beat signal between adjacent pairs at a slightly different frequency so the magnitude and phase between each pair could be resolved by mixing with a swept local oscillator. Mixing two combs with slightly different $\delta f$, for example at 2.4 and $2.5 \mathrm{GHz}$, will produce a series of chirped tones that could be useful as well. Note that the data encoder could be modified to produce tones with a desired frequency spacing in the output spectrum; however, data encoding is typically undertaken over a relatively large block of bits which would produce much lower $\delta f$ than desired for our purposes. Optical phase modulation, discussed in the last section, appears promising for generating a test signal that introduces minimal ISI on the data signal.

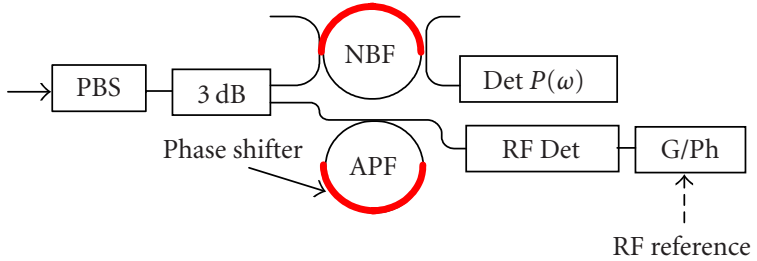

(a)

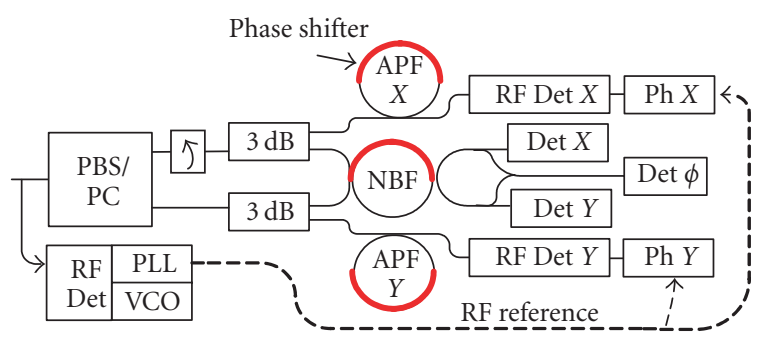

(b)

FIGURE 2: Optical vector spectrum analyzer based on a planar lightwave circuit with ring resonators for (a) a single-input polarization and (b) for measuring PMD.

\section{A NOVEL OPTICAL VECTOR SPECTRUM ANALYZER}

The goal of our OVSA is to determine the amplitude of the tones and relative phase between each pair of tones when they are all present at the detector. While a tunable narrowband filter solves the amplitude discrimination problem, determining the relative phase is more challenging. An obvious solution is to have a bandpass filter with width $\delta f$ or two narrowband optical filters, ring resonators for example, separated by $\delta f$, which scan across the signal spectrum. The relative phase between each pair of tones is then determined via quadrature detection; however, one challenge is having the filters precisely separated as they are tuned, since any undesired offset will contribute phase error arising from the ring dispersion. As the filter is tuned, the tone will be substantially attenuated intermediate to the desired sampling points, which could make the tone difficult to track with a phaselocked loop. Our proposed technique relies on the narrowband phase response of allpass filters instead.

The proposed analyzer circuit for a single-input polarization, which uses a single detector with minimal bandwidth and can easily be realized with planar waveguide ring resonators, is shown in Figure 2a. In this architecture, it is assumed that the input polarization is aligned to a principal state of the device, referred to as the $x$ - or $y$-polarization for a planar waveguide, either by using polarization maintaining components or a polarization controller. For an input signal where information may be carried by either polarization or any combination thereof, simultaneous measurement of orthogonal polarizations is required as shown in Figure 2b. The transfer function of a polarization-dependent system is described by its Jones matrix. In the simplified case of a lossless 
system, the transfer matrix is unitary and described by

$$
\mathbf{M}(\omega)=\left[\begin{array}{cc}
U(\omega) & -V^{*}(\omega) \\
V(\omega) & U^{*}(\omega)
\end{array}\right]
$$

where $U(\omega)$ and $V(\omega)$ are the complex transfer functions for each orthogonal polarization. For a system which is polarization independent or where only a single polarization is important, the transfer function is denoted by $H(\omega)$.

In Figure $2 b$, each polarization is split into a separate path by a polarization beam splitter (PBS) and polarization controller (PC). The PC allows the power in the $x$ - and $y$-outputs to be controlled so that all of the power is not in one output or the other. One polarization on the output of the PBS/PC is rotated by 90 degrees so that the outputs going into the $3 \mathrm{~dB}$ couplers have the same polarization, that is, either $x$ - or $y$-polarization. An integrated polarization beam splitter with a polyimide half-waveplate have been demonstrated [5]. After the $3 \mathrm{~dB}$ coupler, one portion of each polarization is analyzed by a tunable narrowband filter (NBF) to obtain the magnitude across the channel, while the other portion is transmitted through a tunable allpass filter (APF) before being detected. Note that both previously orthogonally-polarized signals experience the same NBF response but in counterpropagating directions, so the polarization dependence of the NBF is not a limiting factor. An additional measurement that is critical for PMD-impaired channels is the relative phase between polarizations [6], which is indicated by the Det $\phi$ in Figure $2 \mathrm{~b}$. The relative phase is measured by tapping off a portion of the $x$ - and $y$-outputs signal and interfering them in a directional coupler. Varying the phase of one of the signals before the coupler, by introducing a phase shifter, allows the relative phase to be determined without ambiguity. Alternatively, a full 90-degree hybrid coupler arrangement as used in coherent detection, where it is used to mix the signal and local oscillator in phase and in quadrature, may be used. The measurement of relative phase and magnitude provides the Jones vector up to a common phase term at each tone.

The filters' resonant frequencies are tuned, for example, by thermo-optic phase shifters. The APFs are identical in principle, but any variations can be compensated via calibration. Each APF is designed to provide a very sharp transition in its phase response from 0 to $2 \pi$ near resonance [7] as shown in Figure 3. On-resonance, the phase is $\pi$. Offresonance, the phase quickly approaches 0 or $2 \pi$. As the resonant frequency is shifted via a phase shifter in the feedback path, the phase response is translated across the channel spectrum and the RF detector records different linear combinations of beats between adjacent tones.

In linear systems terms, the transmission channel for a single polarization has a complex frequency response $H(f)$ which we wish to measure at discrete points defined by the tones. For simplicity, let the tone amplitudes and phases be equal at the transmitter. The response of the system at the $n$th tone is designated by $H(n \delta f)=h_{n} e^{j \varphi_{n}}$, which consists of an amplitude $h_{n}$ and phase $\phi_{n}$. The allpass filter adds an

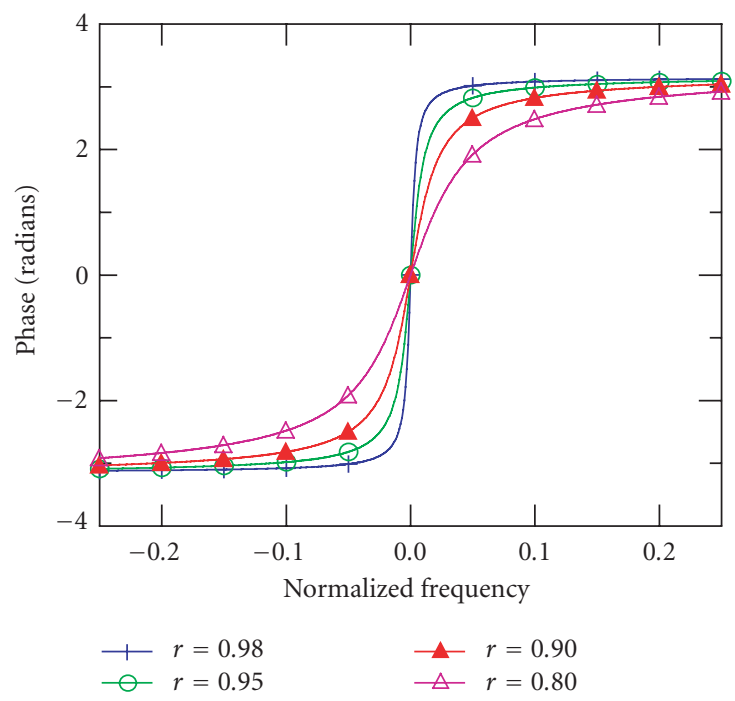

FIgURE 3: A single-stage APF phase response for various values of the pole magnitude.

additional phase term, call it $\theta_{n}$. The detected photocurrent $p(t)$ is proportional to the square magnitude of the field:

$$
p(t) \propto\left|\sum_{-N}^{N} h_{n} e^{j\left(\varphi_{n}+\theta_{n}\right)} e^{j n 2 \pi \delta f t}\right|^{2} .
$$

When the detected photocurrent is mixed with a reference RF signal at the tone difference frequency and lowpass filtered to retain just the $\delta f$ mixing terms, the in-phase and quadrature photocurrent components are given by

$$
\begin{aligned}
& i=\langle p(t) \cos (2 \pi \delta f t)\rangle=\sum_{n=-N}^{N-1} h_{n} h_{n+1} \cos \left(\Delta \varphi_{n+1}+\Delta \theta_{n+1}\right), \\
& q=\langle p(t) \sin (2 \pi \delta f t)\rangle=\sum_{n=-N}^{N-1} h_{n} h_{n+1} \sin \left(\Delta \varphi_{n+1}+\Delta \theta_{n+1}\right),
\end{aligned}
$$

where $\Delta \varphi_{n+1}=\varphi_{n+1}-\varphi_{n}$ and similarly for $\Delta \theta_{n}$. The brackets indicate lowpass filtering by averaging over time. In the ideal case of a lossless APF whose pole magnitude approaches unity, $\Delta \theta_{n}$ approaches 0 or $\pi$ (modulo $2 \pi$ ) and the amplitude response is unity. The phase response becomes more gradual around the resonant frequency as the pole magnitude decreases as shown in Figure 3 for a single-stage APF at several values of the pole magnitude $r$ and for zero frequency offset. Independent of the pole magnitude, the phase at resonance is shifted by $\pi$ relative to the antiresonant frequency $(\omega=+/-\pi)$. The idealized case is assumed in the following discussion for illustrative purposes. In practice, the tone spacing relative to the APF's free spectral range (FSR) determines the pole magnitude required for the phase to change fast enough to make the phase difference approximation by 0 or $\pi$ valid. After the initial explanation, we show how to remove this restriction entirely and also accommodate filters with loss. 

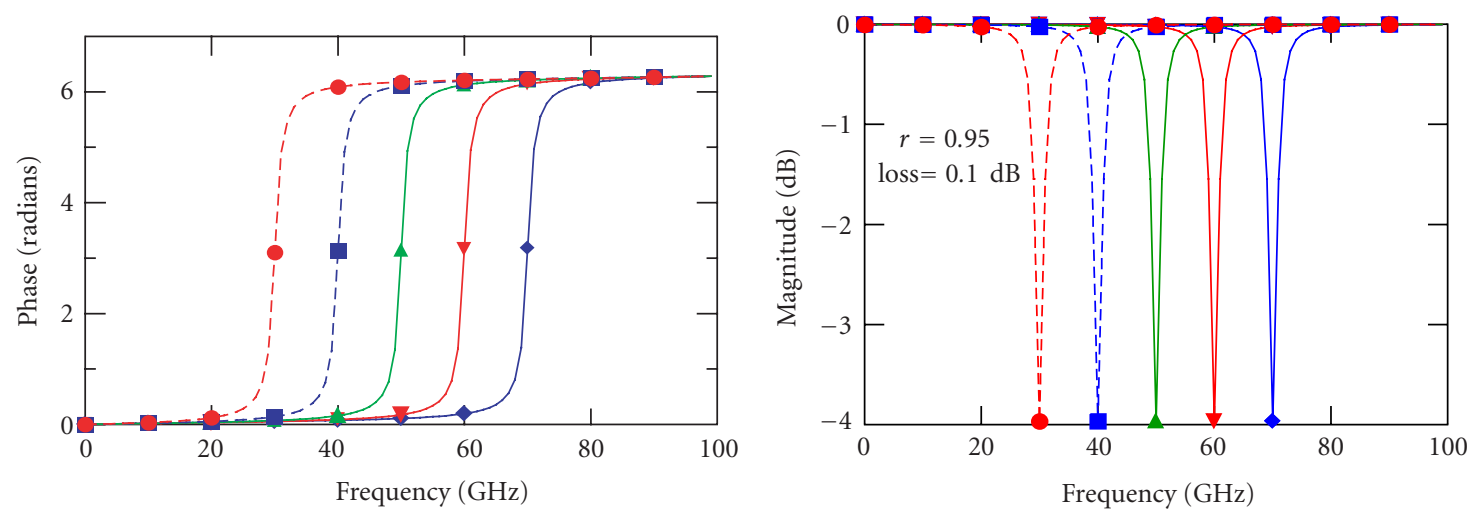

FIGURE 4: Allpass filter phase (left) and magnitude (right) response at several different frequency offsets with the markers indicating the tone locations.

As the APF response is tuned across the tones, the detected $i$ and $q$ change. For the case of a discrete input spectrum, we can represent the phase difference resulting from discrete frequency shifts of an idealized APF response, where zero shift corresponds to the center tone's frequency, using a Kronecker delta function. Let the APF's resonant frequency offset be $m \delta f$ for a particular $i_{m}$ and $q_{m}$ measurement; then the phase difference introduced by an idealized APF (lossless and pole magnitude approaches unity) between the $n$th and $n+1$ st tones is given by $\Delta \theta_{m, n} \approx \pi[\delta(n-m)-\delta(n-1-m)]$ modulo $2 \pi$. Consider a case with three tones. For the first measurement, the APF resonant frequency is set out of band, let $m=-2$, so $\Delta \theta_{0,0}=\Delta \theta_{0,1}=0$ and the quadrature components are

$$
\begin{aligned}
& i_{1}=h_{-1} h_{0} \cos \left(\Delta \varphi_{0}\right)+h_{0} h_{1} \cos \left(\Delta \varphi_{1}\right), \\
& q_{1}=h_{-1} h_{0} \sin \left(\Delta \varphi_{0}\right)+h_{0} h_{1} \sin \left(\Delta \varphi_{1}\right) .
\end{aligned}
$$

For the second measurement, the resonant frequency is set at $f_{0}-\delta f$, or $m=-1$ in this example, so that $\Delta \theta_{1,0}=\pi$ and $\Delta \theta_{1,1}=0$. Then, the quadrature components are

$$
\begin{aligned}
& i_{2}=-h_{-1} h_{0} \cos \left(\Delta \varphi_{0}\right)+h_{0} h_{1} \cos \left(\Delta \varphi_{1}\right), \\
& q_{2}=-h_{-1} h_{0} \sin \left(\Delta \varphi_{0}\right)+h_{0} h_{1} \sin \left(\Delta \varphi_{1}\right) .
\end{aligned}
$$

Thus,

$$
h_{0} h_{1} \cos \left(\Delta \varphi_{1}\right)=\frac{i_{1}+i_{2}}{2}, \quad h_{-1} h_{0} \cos \left(\Delta \varphi_{0}\right)=\frac{i_{1}-i_{2}}{2},
$$

and similarly for the $q$ components so that

$$
\Delta \varphi_{1}=\tan ^{-1}\left[\frac{q_{1}+q_{2}}{i_{1}+i_{2}}\right], \quad \Delta \varphi_{0}=\tan ^{-1}\left[\frac{q_{1}-q_{2}}{i_{1}-i_{2}}\right] .
$$

By detecting $i$ and $q$ at $2 N$ frequency offsets of the APF for an input signal with $2 N+1$ tones, two matrix equations result: $\mathbf{i}=\mathbf{A x}$ and $\mathbf{q}=\mathbf{A y}$, where $\mathbf{x}=\left\{h_{n} h_{n-1} \cos \left(\Delta \varphi_{n}\right)\right\}, \mathbf{y}=$ $\left\{h_{n} h_{n-1} \sin \left(\Delta \varphi_{n}\right)\right\}$, and $\mathbf{A}=\left\{\cos \left(\Delta \theta_{m n}\right)\right\}$ for $-N+1 \leq n$, $m \leq N$. Note that the elements of $\mathbf{A}$ for this ideal case are either 1 or -1 . Magnitude and phase information are given by $h_{n} h_{n-1}=\sqrt{x_{n}^{2}+y_{n}^{2}}$ and $\Delta \varphi_{n}=a \tan \left(y_{n} / x_{n}\right)$, respectively.
In the ideal three-tone case, $\mathbf{A}=\left[\begin{array}{cc}1 & 1 \\ -1 & 1\end{array}\right]$. Once the magnitude response is determined independently at one frequency, then the $\mathbf{x}$ (or $\mathbf{y}$ ) vector is used to calculate the remaining magnitude values.

The APFs provide a functionality that would otherwise have to be obtained by separating the tones into separate paths (i.e., demultiplexing closely spaced tones) and using a phase shifter in each path to obtain the desired series of linear combinations. Note that only the resonant frequency is tuned for both the NBF and APFs, so the coupling ratios are fixed. This allows simple waveguide layouts for the rings, which can achieve very low roundtrip losses. An underlying assumption is that the APF can be translated over $2 N$ points in a time short compared to changes in the system response. An advantage of working with $\delta f^{\prime}$ 's in the range of wireless carrier frequencies, such as $2.5 \mathrm{GHz}$, is that low-cost RF signal processing chips are available. The AD8302 gain and phase measurement circuit by analog devices that operates up to $2.7 \mathrm{GHz}$ is one example. By synchronizing the sweep of the NBF and APFs, the location of the tones in the magnitude response can be used to trigger the sampling of $i$ and $q$. For quadrature detection, a reference signal at the tone frequency is required. This may be obtained by locking a voltage-controlled oscillator (VCO) to the detected RF tone at $\delta f$ using a phase-locked loop (PLL) as shown in Figure 2 in a manner similar to clock recovery on a data signal. Since we are tuning the APF, the tones will never completely drop out during the sweep.

With practical APFs, the pole has a value less than unity, causing the phase to vary more gradually across the resonant frequency region, and the feedback path has some loss, which produces a frequency-dependent amplitude response as shown in Figure 4. The elements of $\mathbf{A}$ are no longer 1 or -1 . The nonidealities of the APF are now included in matrix equations given by

$$
\begin{aligned}
& \mathbf{i}=\mathbf{A x}-\mathbf{B y}, \\
& \mathbf{q}=\mathbf{B} \mathbf{x}+\mathbf{A y},
\end{aligned}
$$

where the elements of $\mathbf{A}$ and $\mathbf{B}$ are given by $a_{n} a_{n-1} \cos \left(\Delta \theta_{m n}\right)$ 

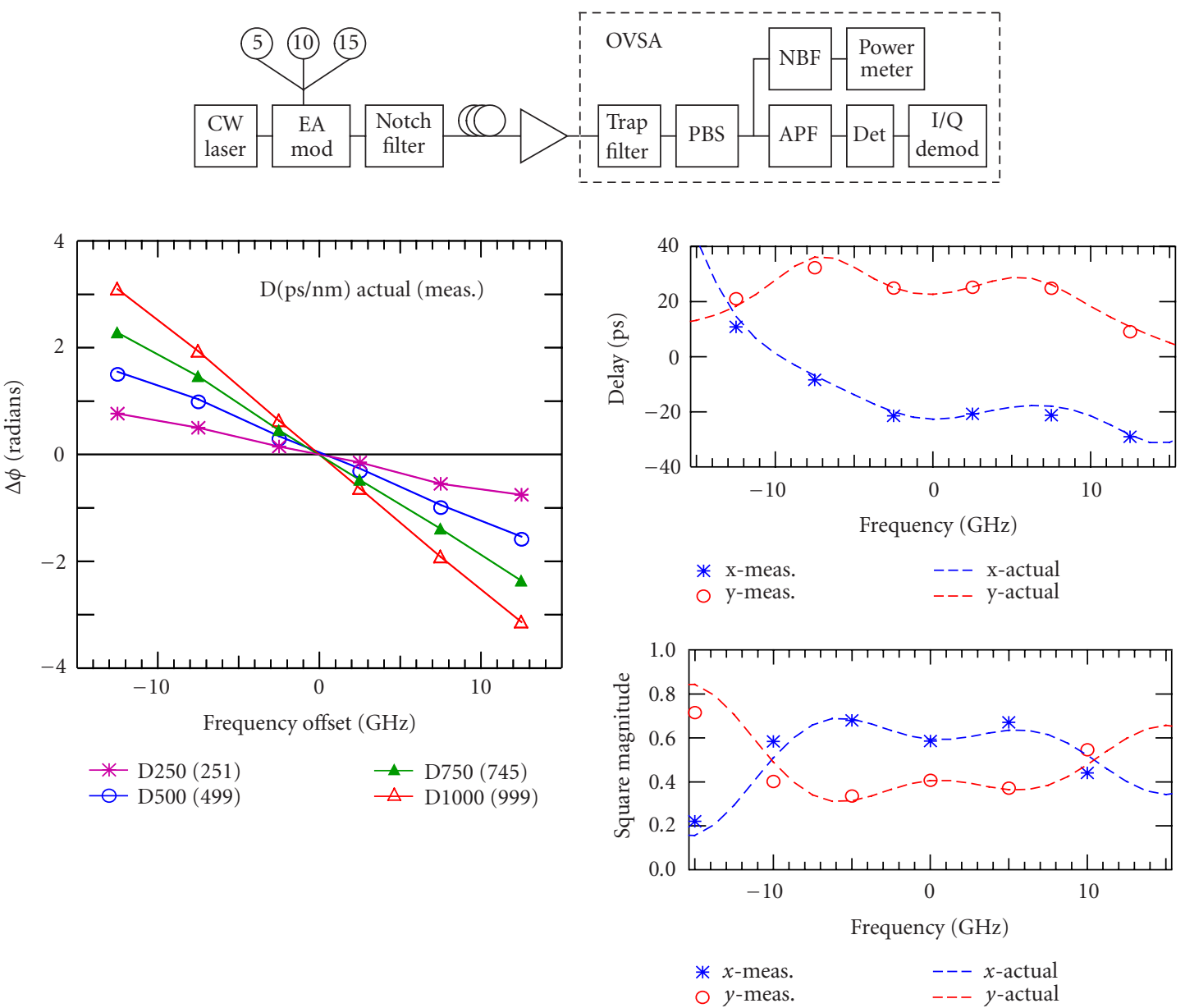

Figure 5: (Top) Simulated system. (Left) Dispersion measurement using 7 tones spaced at $5 \mathrm{GHz}$ and (right) measurement of the Jones matrix frequency responses for a system with PMD.

and $a_{n} a_{n-1} \sin \left(\Delta \theta_{m n}\right)$, respectively, and the magnitude response of the APF at the tone frequencies is represented by the $a_{n}$ terms. Note that $\mathbf{A}$ and $\mathbf{B}$ depend only on the APF response and not the system, so they can be determined by a one-time calibration procedure. Formula (8) can be rearranged to solve for $\mathbf{x}$ and $\mathbf{y}$ as follows:

$$
\begin{gathered}
\mathbf{x}=\mathbf{C}^{-1}\left(\mathbf{B}^{-1} \mathbf{i}+\mathbf{A}^{-1} \mathbf{q}\right), \\
\mathbf{y}=\mathbf{C}^{-1}\left(-\mathbf{A}^{-1} \mathbf{i}+\mathbf{B}^{-1} \mathbf{q}\right),
\end{gathered}
$$

where $\mathbf{C}=\left(\mathbf{A}^{-1} \mathbf{B}+\mathbf{B}^{-1} \mathbf{A}\right)$. The requirement for the tunable APF is that it sample the tones in a manner so that $\mathbf{A}$ and $\mathbf{B}$ are nonsingular and therefore invertible. This condition is certainly met with nonideal, but practical, parameters for the APF as shown in Figure 4 of $0.1 \mathrm{~dB} /$ roundtrip loss and the pole at 0.95 . When the input tones occur mainly on one side or the other of the allpass filter's resonant frequency, the APF's response is dominantly quadratic in phase and it acts like chromatic dispersion from an optical fiber. It is well known that chromatic dispersion, which is characterized by a quadratic phase response, causes fading of RF tones [8].

\section{SYSTEM SIMULATIONS}

System simulations using commercial software ${ }^{1}$ were first run using a comb produced by an electro-absorption modulator driven by 3 frequencies at 5, 10, and $15 \mathrm{GHz}$ (Figure 5). A notch filter was used to attenuate the carrier by $20 \mathrm{~dB}$ so that all tones had approximately the same magnitude. The output power after the notch filter was $-24 \mathrm{dBm}$. The relative phase between each pair of adjacent tones was calculated and is plotted in Figure 5 for various cumulative system dispersions. The linear fit for the measured dispersion is within $1 \%$ of the actual. Then, the frequency response of a system with simulated PMD, consisting of a cascade of 1000 birefringent plates with random orientations, was tested. The results are shown for both the delay and magnitude in Figure 5. The group delay difference between orthogonal polarizations varies across the channel and has a value of 45 picoseconds at the center frequency. The dominant noise source was outof-band tones generated from nonlinearities in the modulator. A trapezoidal filter was used to attenuate these unwanted

\footnotetext{
${ }^{1}$ VPItransmissionMaker by Vitual Photonics, Inc.
} 


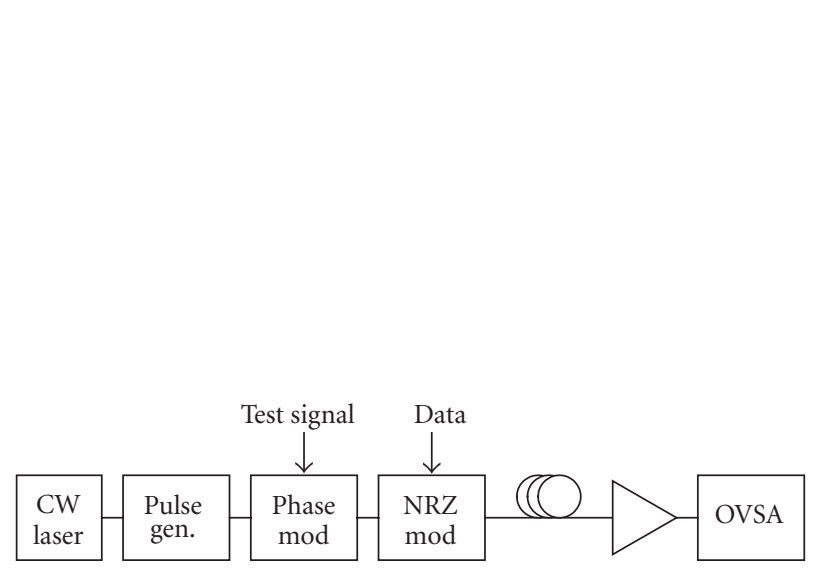

(a)

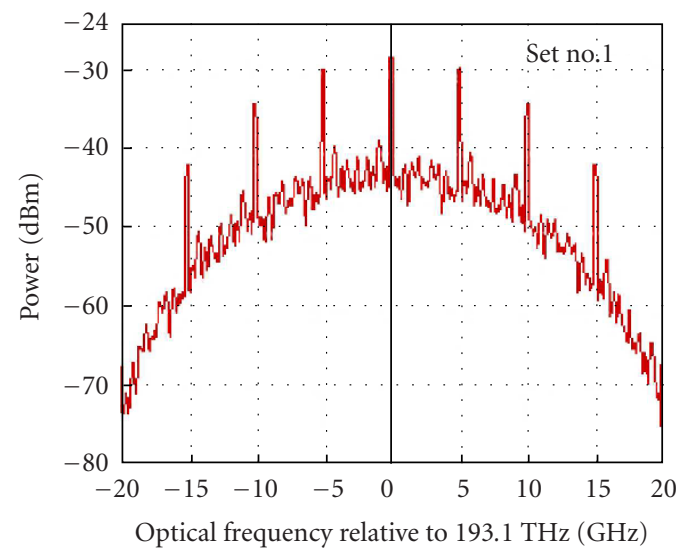

(c)

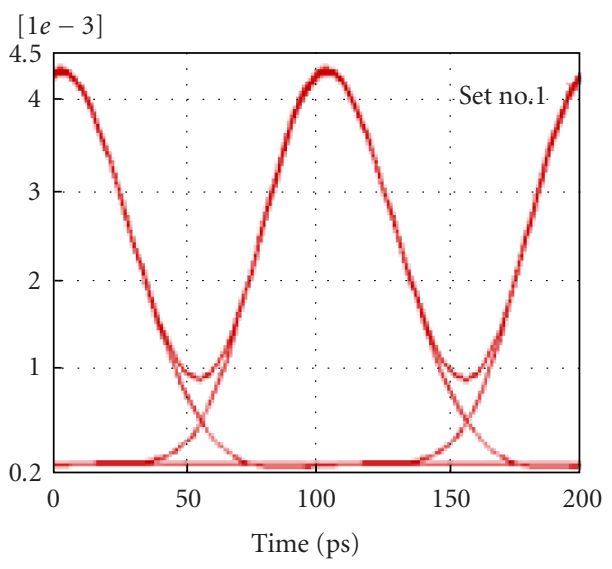

(b)

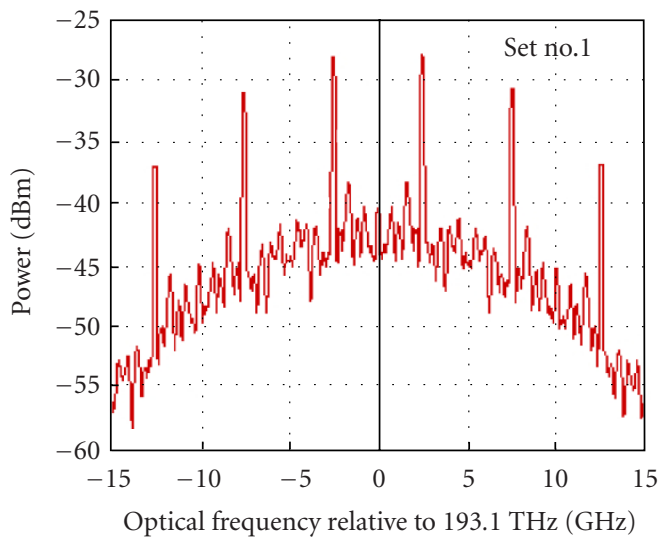

(d)

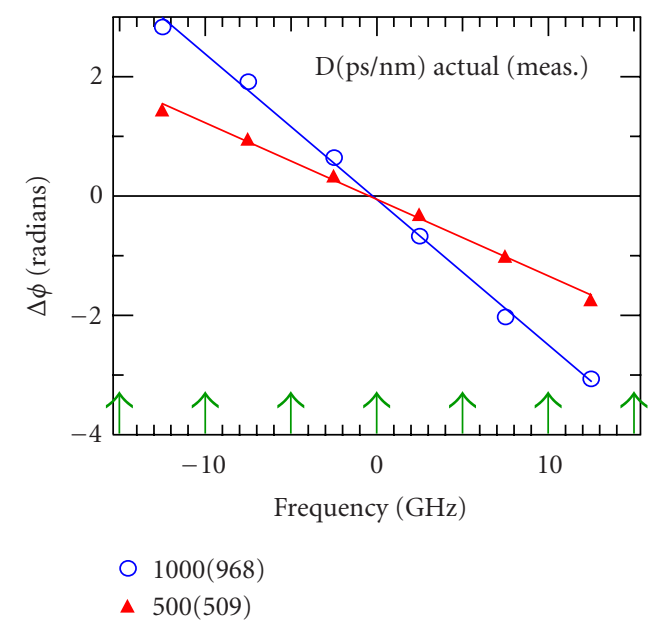

(e)

Figure 6: (c), (d) An RZ 33\% duty cycle signal phase modulated to produce tones at half the bitrate, and (e) measured dispersion. (a) The system simulation schematic and (b) the transmitted eye diagram are shown.

tones by at least $20 \mathrm{~dB}$. A 7th-order bandpass Bessel filter centered at the tone frequency was placed after the detector and before the quadrature demodulation circuit. The input power to the optical amplifier was reduced to increase the 
amplified spontaneous emission noise, but it did not impact the measurements, which illustrates an advantage of using narrowband quadrature detection.

In practice, we wish to have the test and data signals coexist. For amplitude modulation and direct detection, phase modulation represents an orthogonal space for introducing the test signal, as shown in Figure 6. An RZ pulse generator was followed by a phase modulator with the repeating pattern $[1010] \pi / 2$ and then by an NRZ amplitude modulator for the signal (PRBS in this case) to produce the spectrum in Figure $6 \mathrm{c}$. The resulting test signal is orthogonal to the data using direct detection so it does not introduce ISI. By varying the pattern, which is effectively at half the bitrate in this example, and modulation depth, the tone locations can be translated in frequency. A phase modulation of [1100] $\pi$ produced the spectrum in Figure $6 \mathrm{~d}$. Simulated dispersion measurements with $3 \%$ or less error from the actual dispersion are shown in Figure 6e for a single polarization, $10 \mathrm{Gbp} / \mathrm{s} \mathrm{RZ}$ (33\% duty cycle) signal with a $24 \mathrm{~dB}$ OSNR. Similar results to Figure 5 were also obtained for a system with PMD.

If there is no dispersion in the system, the RF spectrum has tones only at the clock rate. To obtain a reference signal for quadrature detection at half the bitrate, either the clock can be recovered and halved, for example by a divide-by2 circuit, or APFs can be introduced as in the analyzer circuit itself to break the deconstructive interference condition between adjacent tones under direct detection and produce a detectable RF tone. Another practical consideration is the phase calibration. The relative phases between adjacent tones at the transmitter must be known. A back-to-back measurement was used as the calibration for these simulations.

For polarization multiplexed signals, a slightly different comb frequency difference $\delta f$ can be used for each polarization and retrieved by mixing with the appropriate local oscillator after detection. Alternatively, the tones can be staggered as indicated in Figures $6 \mathrm{c}$ and $6 \mathrm{~d}$. Let the Jones vector at the input for each polarization be denoted by $\mathbf{S}_{X}=$ $\left[\begin{array}{ll}S_{X}(\omega) & 0\end{array}\right]^{T}$ and $S_{Y}=\left[\begin{array}{ll}0 & S_{Y}(\omega)\end{array}\right]^{T}$, where $S(\omega)$ is the input spectrum. The output, measured by a circuit such as shown in Figure 2, is then given by $\mathbf{H}=\mathbf{M}\left(\mathbf{S}_{X}+\mathbf{S}_{Y}\right)$, where $\mathbf{H}=\left[H_{X}(\omega) H_{Y}(\omega)\right]^{T}$, and

$$
\begin{aligned}
& H_{X}(\omega)=U(\omega) S_{X}(\omega)-V^{*}(\omega) S_{Y}(\omega) \\
& H_{Y}(\omega)=V(\omega) S_{X}(\omega)+U^{*}(\omega) S_{Y}(\omega)
\end{aligned}
$$

Thus, it is possible to measure the system Jones matrix with this technique without varying the input polarization to the system. For PMD compensation, this provides the information needed to estimate the ideal compensating function, $\mathbf{M}^{-1}(\omega)$.

In summary, a new technique was presented for obtaining phase and magnitude information from a signal using tunable, optical allpass filters. While a particular application was cited, the underlying approach of applying allpass filtering to obtain spectrally dependent phase information without optical heterodyning or without bandlimiting the signal in the optical domain is general. Low-coherence techniques allow one to determine the phase of a device embedded in an interferometer; however, the proposed technique allows one to obtain phase information on the source and, subsequently, of the device or system. Optical modulation is proposed to introduce a test signal that is continuous in time and does not introduce ISI. Channel estimation at discrete frequency points across a noisy signal spectrum was demonstrated via simulation; thus, the available information for controlling PMD compensators and polarization demultiplexers is substantially enhanced. The technique is also applicable to characterizing a periodic pulse stream with an arbitrary waveform shape. The first experimental results for characterization of a periodic pulse stream were reported in [9].

\section{REFERENCES}

[1] C. K. Madsen, "Optical all-pass filters for polarization mode dispersion compensation," Optics Letters, vol. 25, no. 12, pp. 878-880, 2000.

[2] A. Eyal and A. Yariv, "Design of broad-band PMD compensation filters," IEEE Photon. Technol. Lett., vol. 14, no. 8, pp. 10881090, 2002.

[3] C. K. Madsen, "Chromatic and polarization mode dispersion measurement technique using phase-sensitive sideband detection," in Proc. Optical Fiber Communication Conference (OFC '01), vol. 1, pp. MO6-1-MO6-3, Anaheim, Calif, USA, March 2001.

[4] L. Moller and L. Buhl, "Method for PMD vector monitoring in picosecond pulse transmission systems," J. Lightwave Technol., vol. 19, no. 8, pp. 1125-1129, 2001.

[5] T. Saida, Y. Orihara, H. Yamada, K. Takiguchi, T. Goh, and K. Okamoto, "Integrated optical polarisation analyser on planar lightwave circuit," Electronics Letters, vol. 35, no. 22, pp. 19481949, 1999.

[6] C. K. Madsen, E. Laskowski, J. Bailey, et al., et al., "An integrated wavelength-sensitive polarimeter," in Proc. European Conference on Optical Communications (ECOC '02), Copenhagen, Denmark, September 2002.

[7] C. K. Madsen and J. Zhao, Optical Filter Design and Analysis: A Signal Processing Approach, John Wiley, New York, NY, USA, 1999.

[8] B. Christensen, J. Mark, G. Jacobsen, and E. Bodtker, "Simple dispersion measurement technique with high resolution," Electronics Letters, vol. 29, no. 1, pp. 132-134, 1993.

[9] C. K. Madsen, M. Cappuzzo, E. Chen, et al., "A novel optical vector spectrum analysis technique," in Conference on Lasers and Electro-Optics (CLEO '04), San Francisco, Calif, USA, May 2004, Postdeadline CPDD5.

C. K. Madsen, a Professor of electrical engineering at Texas A\&M University, College Station, IEEE Senior Member, and an OSA Fellow, received the B.S. degree from the University of Texas at Austin in 1986, the M.S. degree from Stanford University, Standford, Calif, in 1987, and the Ph.D. degree from Rutgers University, Piscataway, NJ, in 1996, all in electrical engineering. She joined AT\&T Bell Laboratories in 1987 and worked for the submarine systems business unit. After completing her Ph.D., she transferred to the Integrated Photonics Research Department at Bell Laboratories. Since then, her research has focused on the application of digital filter and signal processing techniques 
to optical filters for high-speed, high-capacity optical communication systems. In 1998, Madsen invented a class of tunable, multistage optical allpass filters that allow any phase response to be approximated and have application in chromatic dispersion compensation and polarization-mode dispersion compensation. She has given a short course on "Optical Filters for WDM Systems: Theory, Technologies, and Applications" at OFC and was the 2004 General Chair for the Integrated Photonics Research (IPR) Conference. She was promoted to Distinguished Member of the Technical Staff at Bell Laboratories in 2002 and achieved a Fellow ranking in the Optical Society of America in 2003. She holds 16 US patents and has given over 70 technical talks and papers. In 2004, she joined Texas A\&M University as a Full Professor. 